\title{
PREDICTIONS OF CIRCULATING CURRENT FIELD AROUND A SUBMERGED BREAKWATER INDUCED BY BREAKING WAVES AND SURFACE ROLLERS
}

\author{
Yoshimitsu Tajima ${ }^{1}$
}

\begin{abstract}
This paper develops a quasi-three-dimensional nearshore current model which accounts for excess shoreward volume fluxes due to waves and surface rollers. The model splits the water column at the wave trough level and solves two different layers of 2DH momentum equations. The first layer covers above the trough level and determines the shear stress at the trough level while the the second layer covers over the entire depth and determines the volume flux due to mean current components. Linearly approximated vertical profile of the shear stress and corresponding turbulent eddy viscosity model enables the present model to yield analytical explicit expression of the horizontal velocity profiles, which enhance computational efficiency of the model. The extended model was applied to predictions of longshore current velocity on the long straight beach and circulation current patterns behind the detached submerged breakwater on the plane beach. Excellent agreement between model predictions and measurements supports the validity of the model and importance of quasi-three-dimensional features around the submerged break water.
\end{abstract}

Keywords: submerged breakwater, Q3D nearshore current model, surface roller, wave breaking

\section{INTRODUCTION}

After the revision of the coastal law in 1999 in Japan, a submerged breakwater became one of preferred options as coastal protection measures. A submerged breakwater has advantages in that it has less impact on the coastal landscapes and it also enhances water exchanges to keep the water quality behind the structure. Submerged-type breakwater however tends to cause more complicated circulation current patterns and, as a result, these structure often failed to realize the expected coastal protection functions. For example, breaking and broken waves on the submerged structure should transport excess amount of water toward the shore over the structure. The excess amount of shoreward water increases mean water level behind the structure and may alter the forcing balances of the circulating current field behind the breakwater. Excess amount of shoreward volume flux may also enhance the returning undertow velocity near the water bottom. Widely used two-dimensionalhorizontal (2DH) nearshore current model cannot properly express such phenomena. In addition to such three-dimensional phenomena, submerged-type breakwater may cause complex wave field due to breaking, reflection and diffractions of waves.

Time-dependent non-linear dispersive wave model such as Boussinesq-type model may be one of preferred models to directly express such complex wave-current co-existing field. Tajima et al (2007), for instance, applied Boussinesq-type model for predictions of the circulation current around the submerged breakwater and pointed out that the predictive skills of the circulation current are strongly dependent on the breaking wave models and most models fail to represent the observed circulation current patterns. Besides breaking model, depth-integrated two-dimensional-horizontal (2DH) timedependent model also has drawbacks in that the model accounts only for the wave-associated vertical profiles of the horizontal current velocity and does not compute the vertical profiles of the mean shear current velocity. Furthermore, the model does not account for the shoreward volume flux due to broken waves and surface rollers, which should have significant influence on the return flow velocity. Especially around the submerged breakwater, broken waves appear to transport extensive amount of water toward the shore near the water surface and the model may underestimate the return flow velocity under the wave trough level, which compensates for the shoreward volume flux.

As a model that keeps computational efficiency and capability of accounting vertical profiles of horizontal shear current velocity, quasi-three-dimensional (Q3D) models have been developed by various researchers. Some of these Q3D model directly computes horizontal velocity at arbitral elevation under the approximating assumption of hydrostatic pressure (e.g., Kuroiwa et al., 1997). Most of other Q3D models determine approximate vertical profile of the horizontal velocity, substitute it into the horizontal momentum equations and then integrate the obtained momentum equations in

\footnotetext{
${ }^{1}$ Department of Civil Engineering, The University of Tokyo, Hongo 7-3-1, Bunkyo-ku, Tokyo, 113-8656, Japan
} 
the vertical direction to yield the depth-integrated 2DH momentum equations (e.g., Svendsen and Putrevu, 1994, Sanchez et al., 1992). Similar to the well-known 2DH nearshore current model, most of these Q3D models apply the phase-averaged momentum equations with wave radiation stress and dispersion terms with empirically determined dispersion coefficients. Svendsen and Putrevu (1994) on the other hand pointed out in their model, SHORECIRC that extra advection terms which are yielded by accounting for vertical variation of the horizontal current velocity, have similar effect of horizontal dispersion terms.

Tajima and Madsen's (2005) undertow model can be applied for estimations of vertical profiles of both undertow and longshore current velocity when periodic or random waves were obliquely incident on a long straight beach. Besides advection effects similar to SHORECIRC, the model accounts for the influence of surface rollers both in momentum and mass conservation equations. Influence of excess amount of volume flux due to broken waves and surface rollers may be one of crucial factors for better predictions of the mean shear current field behind the submerged breakwater. This study thus aims to extend Tajima and Madsen's (2005) model to the arbitrary Q3D problems and eventually apply it to the predictions of current field around the submerged breakwater.

\section{MODEL DESCRIPTIONS}

The entire model consists of three sub-models for predictions of: (i) wave shoaling and breaking, (ii) surface roller evolution and (iii) Q3D nearshore current field. This study applies Tajima and Madsen's (2006) broken wave and surface roller models. The Q3D nearshore current model inputs phase-averaged forces and volume fluxes associated with waves and surface rollers. Figure 1 illustrates the basic concept of the present model. As shown in the Figure, the model splits the water column into two parts at the wave trough level and determine the 2DH phase-averaged momentum equations vertically integrated over the two different layers: one above the wave trough level; and the other over the entire depth. More details of the model are discussed in the following sections.

\section{Governing Equations}

Under the wave trough level, vertical gradient of the horizontal velocity is determined by following momentum equation.

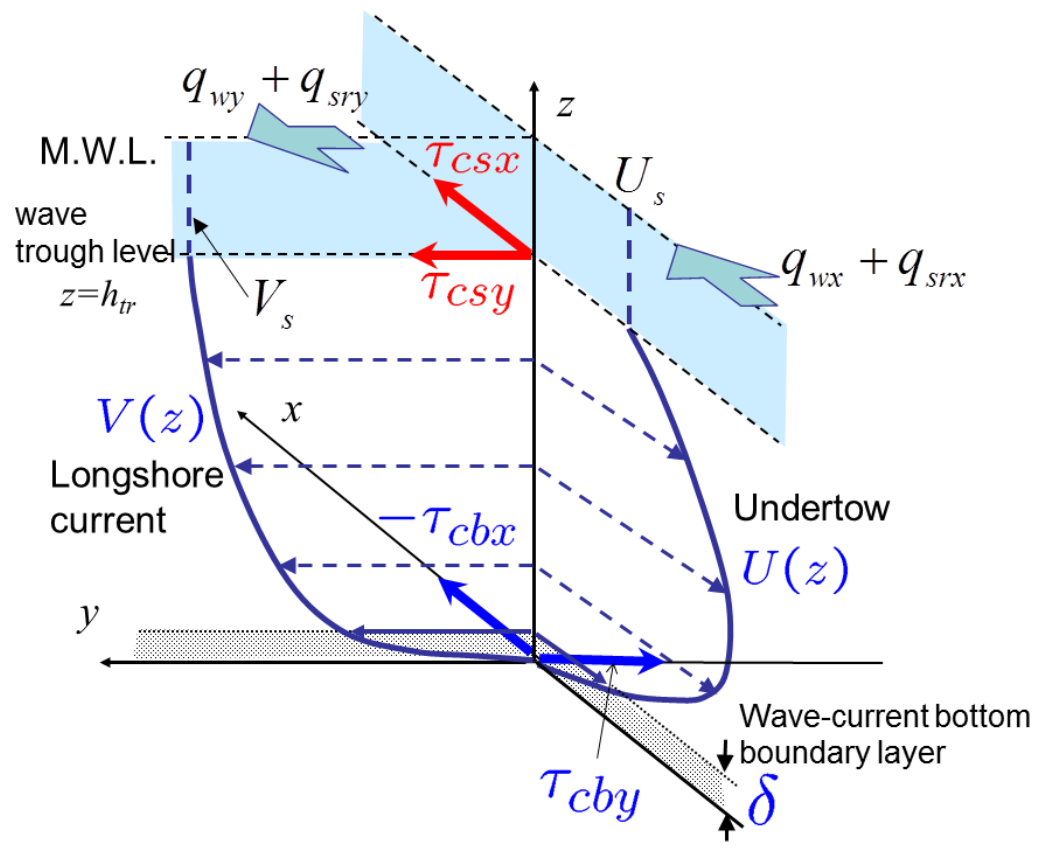

Figure 1. Definitions of the variables used in the model. 


$$
\rho v_{T} \frac{\partial U_{i}}{\partial z}=\tau_{c z, i}=\tau_{c b, i}+\frac{\tau_{c s, i}-\tau_{c b, i}}{h_{t r}} z
$$

where $U_{i}$ is horizontal velocity components in the $x_{i}$ direction $(i=1,2), \rho$ is water density, $v_{\mathrm{T}}$ is turbulent eddy viscosity discussed later, $\tau_{c z, i}$ is phase averaged horizontal shear stress acting in the $x_{i}$ direction, $\tau_{c b, i}$ and $\tau_{c s, i}$ are horizontal shear stress respectively at bottom and trough level and $h_{t r}$ is the water depth under the trough level. Vertical profile of the shear stress distributions is approximately assumed to be linear. This assumption is valid if the wave-induced forces including surface rollers are dominant under the shallow water approximations. Linear approximation moreover enables us to determine the shear stress at arbitrary elevation only from the shear stresses at bottom and at wave trough level.

The present turbulent eddy viscosity model follows the one proposed by Tajima and Madsen (2006), in which, besides general law-of-the-wall-type linear eddy viscosity model, influence of excess turbulence caused by broken waves and by shear stress acting at the wave trough level is accounted for. Combined with determined turbulent eddy viscosity model, equation (1) enables us to determine the vertical profile of the horizontal current velocity in an analytical expression, which significantly improves the computational efficiency of the model. Finally, as seen in Figure 1, mean current velocity above the trough level was simply assumed to be constant with their quantities represented by those determined at the wave trough level.

The shear stress at the trough level, $\tau_{c s, i}$ is determined by integrating horizontal momentum equation from the surface to the wave trough level:

$$
\tau_{c s, i}=F_{s h p, i}+F_{s w, i}+F_{s r, i}+F_{s c, i}+F_{s w c, i}+F_{s r c, i}+F_{s v, i}
$$

where the seven terms in the right hand side of equation (2) are respectively: (i) hydrostatic pressure force; (ii) momentum force due to wave motion; (iii) momentum force due to surface roller; (iv) momentum force fucurrent-current interaction; (v) momentum force due to wave-current interactions; (vi) momentum force due to current-surface roller interactions; and (vii) horizontal shear stress due to turbulence. Detailed expressions of each term are as follows.

$$
\begin{gathered}
F_{s h p, i}=-\rho g a \frac{\partial \bar{\eta}}{\partial x_{i}} \\
F_{s w, i}=-\frac{\partial}{\partial x_{i}}\left(\frac{E}{2}\right)-\frac{\rho}{2}\left\{a \hat{u}_{s, j} \frac{\partial \hat{u}_{s, i}}{\partial x_{j}}-a \frac{\partial \hat{w}_{s}^{2}}{\partial x_{i}}-\hat{w}_{s}^{2} \frac{\partial \bar{\eta}}{\partial x_{i}}\right\} \\
F_{s r, i}=-\frac{\partial}{\partial x_{j}} R_{j i} \\
F_{s c, i}=-\rho a U_{s, j} \frac{\partial U_{s, i}}{\partial x_{j}} \\
F_{s w c, i}=-\frac{\rho a}{2}\left(\hat{u}_{s, j} \frac{\partial U_{s, i}}{\partial x_{j}}+U_{s, j} \frac{\partial \hat{u}_{s, i}}{\partial x_{j}}\right) \\
F_{s v, i}=\rho a \frac{\partial}{\partial x_{j}}\left(v_{T s} \frac{\partial U_{s, i}}{\partial x_{j}}\right) \\
\left.C F_{s c, i} \frac{\partial U_{s, i}}{\partial x_{j}}-U_{s, j} \frac{\partial C n_{i}}{\partial x_{j}}\right) \\
C T\left(\begin{array}{l}
C n_{j} \\
F_{j}
\end{array}\right)
\end{gathered}
$$


where $\bar{\eta}$ is mean water level, $E$ is wave energy, $\hat{u}_{s, i}$ and $\hat{w}_{s}$ are amplitude of the wave-associated oscillating current velocity at the surface respectively in the $x_{i}$ and $z$ directions, $R_{i j}$ is "radiation stress" of the surface roller, $U_{s, i}$ is the mean current velocity components in the $x_{i}$ direction, $A_{s r}$ is the crosssectional area of the surface roller per each wave length, $T$ is wave period, $C$ is wave phase velocity, $n_{i}$ is a $x_{i}$-component of the unit vector of the wave propagating direction, $v_{\mathrm{Ts}}$ is a turbulent eddy viscosity near the surface and $j=1,2$ follows the summation convention. Svendsen and Putrevu's (1994) model does not account for the third, fifth, and sixth terms in equation (2), i.e., the momentum forces due to surface roller, wave-current and roller-current interactions. Surface roller radiation stress tensor, $R_{i j}$, and the cross-sectional area of the roller, $A_{s r}$, are respectively determined by

$$
\begin{gathered}
R_{i j}=\frac{\rho A_{s r} C^{2} n_{i} n_{j}}{C T}=2 E_{s r} n_{i} n_{j} \\
A_{s r}=\frac{2 T E_{s r}}{\rho C}
\end{gathered}
$$

Similar to the shear stress at the trough level, the shear stress at the bottom can also be determined by integrating the horizontal momentum equation over the entire water depth, i.e.,

$$
\tau_{c b, i}+\rho \frac{\partial q_{c, i}}{\partial t}=F_{b h p, i}+F_{b w, i}+F_{s r, i}+F_{b c, i}+F_{b w c, i}+F_{b s r c, i}+F_{b v, i}
$$

Respective contributing factors of the forcing terms on the right hand side of equation (5) are the same as the ones in equation (2) while their expressions are slightly different since the integration range of the momentum equation is different.

$$
\begin{gathered}
F_{b h p, i}=-\rho g h \frac{\partial \bar{\eta}}{\partial x_{i}} \\
F_{b w, i}=-\frac{\partial S_{j i}}{\partial x_{j}} \\
F_{b c, i}=-\rho \frac{\partial}{\partial x_{j}}\left(a U_{s, j} U_{s, i}+q_{b, j} U_{0, i}\right) \\
F_{b w c, i}=-\frac{\rho}{2} \frac{\partial}{\partial x_{j}}\left(a \hat{u}_{s, j} U_{s, i}+a U_{s, j} \hat{u}_{s, i}\right) \\
F_{b s r c, i}=-\frac{\rho}{T} \frac{\partial}{\partial x_{j}}\left\{A_{s r}\left(n_{j} U_{s, i}+n_{i} U_{s, j}\right)\right\} \\
F_{b v, i}=\rho h \frac{\partial}{\partial x_{j}}\left(v_{T} \frac{\partial U_{s, i}}{\partial x_{j}}\right)
\end{gathered}
$$

where $S_{i j}$ is well-known wave radiation tensors, $q_{b i}$ is mean volume flux component under the trough level in the $x_{i}$ direction, $U_{0}$ is averaged mean current velocity under the trough level and $v_{\mathrm{T} 0}$ is depthaveraged turbulent eddy viscosity. Introduction of these two depth-averaged values assumes that fluctuations of mean current velocity in the vertical direction are small relative to its depth-averaged velocity.

As seen in equation (5), the model keeps a time-derivative term of $q_{c, i}$, depth-integrated volume flux due to mean current components. Note that phase-averaged velocity components of waves and surface rollers should be constant in time and thus the time-derivative term was only left for the 
depth-integrated mean current components. While the present model aims to determine the steady state conditions, the model keeps time-derivative term in equation (5) and, as discussed in the next section, the model uses this time-derivative term to iteratively estimate the modification of the volume flux, $q_{c, i}$, until it reaches to the steady state conditions.

Tajima and Madsen (2006) pointed out that all of these phase-averaged terms related to wave and surface roller motions are determined as functions of phase-averaged energy of the waves and surface rollers and thus the wave model can be a simple and computationally efficient phase-averaged-type model such as energy balance equations. In this study, however, we apply time-dependent linear mild slope equations for evaluations of the wave field since the partial reflected waves around the submerged break water may play significant role to determine the phase-averaged forcing field.

As discussed in the previous paragraph, depth-integrated 2DH momentum equation (5) is used to determine the volume flux of the mean current component, $q_{c, i}$ and the model still needs to determine the bottom shear stress and mean water level. To estimate the bottom shear stress under wave current coexisting field, the present model followed Tajima and Madsen's (2006) model. Tajima and Madsen's (2006) model, based on Madsen's (1994) wave-current bottom boundary layer model, determines the phase averaged bottom shear stress as a function of: (i) amplitude of near-bottom wave orbital velocity; (ii) wave period; (iii) angle between wave and mean shear stress; (iv) equivalent bottom roughness; (v) depth-integrated volume flux, $q_{c, i}$. Finally, mean water level is determined by application of the depth-integrated mass conservation equation, i.e.,

$$
\frac{\partial \bar{\eta}}{\partial t}=-\frac{\partial}{\partial x_{j}}\left(q_{c, j}+q_{w j}+q_{s r, j}\right)
$$

\section{Computation Procedures}

The present model differs from the Tajima and Madsen's (2006) undertow model in that the model determines the mean water level from the time-dependent mass conservation equation. Numerical computation procedures of the present Q3D nearshore current model are summarized as follows:

1. Set mean water level, $\bar{\eta}$, mean current velocity components, $U_{s i}$, and $U_{0 i}$ to be zero as initial conditions.

2. Compute $q_{c i}$ of the newer time step from the discretized equation of (5). Gradually increase waveassociated and surface roller-associated terms from zero to the actual values as the numerical iterations proceed.

3. Estimate mean water level from (6) with newly estimated $q_{c, i}$.

4. Apply Tajima and Madsen's (2006) bottom boundary layer model and estimate bottom shear stress as functions of newly determined $q_{c i}$.

5. Replace former variables by newly estimated ones and return back to the number, 2, and iterate the same procedures until estimated $q_{c i}$ and $\tau_{c b, i}$ reach to the equilibrium state.

\section{MODEL APPLICATIONS}

The present extended Q3D model was applied to two different cases: the first case is when periodic or random waves were obliquely incident on the long straight beach; and the second case is when periodic waves were normally incident on the plane beach with submerged detached breakwater installed.

\section{Longshore Currents and Undertow Velocity Profiles on the Long Straight Beach}

The present model was first applied to the experimental case presented by Hamilton and Ebersole (2001), who utilized their Large-Scale Sediment Transport Facility (LSTF) to generate the longshore current when periodic or random waves were obliquely incident on the long straight beach. Figure 2 and Figure 3 compare predicted and measured wave heights, wave setup, vertical profiles of crossshore and longshore mean current velocity, and cross-shore distributions of the depth-averaged longshore current velocity for the experimental cases of Test $6 \mathrm{~A}-\mathrm{N}$ and $8 \mathrm{~A}-\mathrm{E}$, respectively. Test $6 \mathrm{~A}-\mathrm{N}$ applied regular wave while Test $8 \mathrm{~A}-\mathrm{E}$ applied random wave. In the figure, wave heights were 
computed by energy balance equation with breaking and broken dissipation model presented by Tajima and Madsen (2006). In Figure 3, dashed line shows the computed results when the regular wave conditions were assumed instead of random waves. Excellent agreements of these comparisons surely validate the predictive skills of the present model not only for wave properties but also for cross-shore and longshore current velocities both under regular and random wave cases.

\section{Phase-Averaged Current Field around the Detached Submerged Breakwater}

The present model was finally applied to the case when regular waves were normally incident on the plane beach with submerged breakwater. Figure 4 shows overview of the experimental conditions performed by Tajima et al. (2007). As seen in the figure, this experiment introduces vertical walls on both sides of the plane beach and represents the long straight beach conditions with the mirror-images reflected at the walls. In addition, steeply sloping 1:1 edge of the submerged break water should cause partial reflections. Under such conditions, reflected wave components should have significant influence on the nearshore current field and thus phase-averaged energy balance equation, which cannot account for wave phase information, may not be the optimum wave model to be applied in this specific case. This study therefore applied linear mild slope equations (Oonaka and Watanabe, 1987) to determine the wave and surface roller field.

Following Tajima and Sato (2010), Tajima and Madsen's (2006) broken wave model was introduced to the mild slope equations so that wave attenuation obtained in this model should be consistent with that of the energy balance equation model. As discussed in the previous section, the present Q3D model requires inputs of phase-averaged wave properties such as wave radiation stress and volume flux due to wave motions. Since the time-dependent mild slope equation yields instantaneous surface water levels, $\zeta$, and horizontal velocities, $u_{i}$, and volume flux, $P_{i}$, this study followed Tajima and Sato (2010) and numerically computed phase-averaged radiation stress tensor, $S_{i j}$, and wave-induced volume flux $q_{w i}$, by

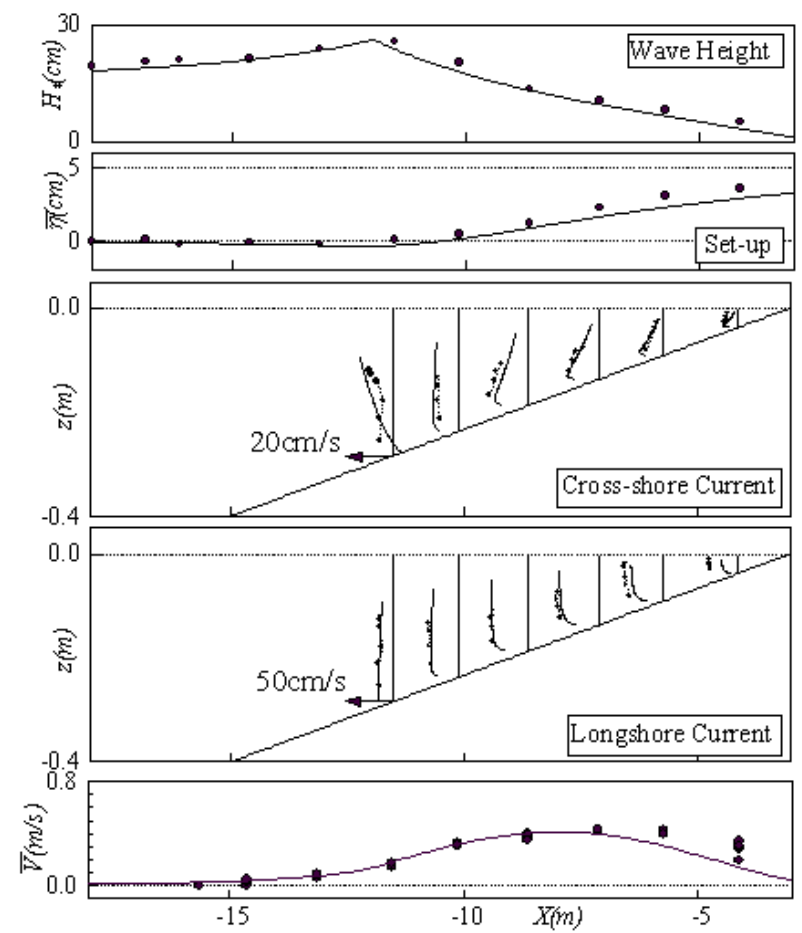

Figure 2. Comparisons of computed wave heights, setup, vertical profiles of cross-shore and longshore current velocities, cross-shore distributions of depth-averaged longshore current velocities with measured data presented by Hamilton and Ebersole (2001) (Test 6A-N, regular wave case). 


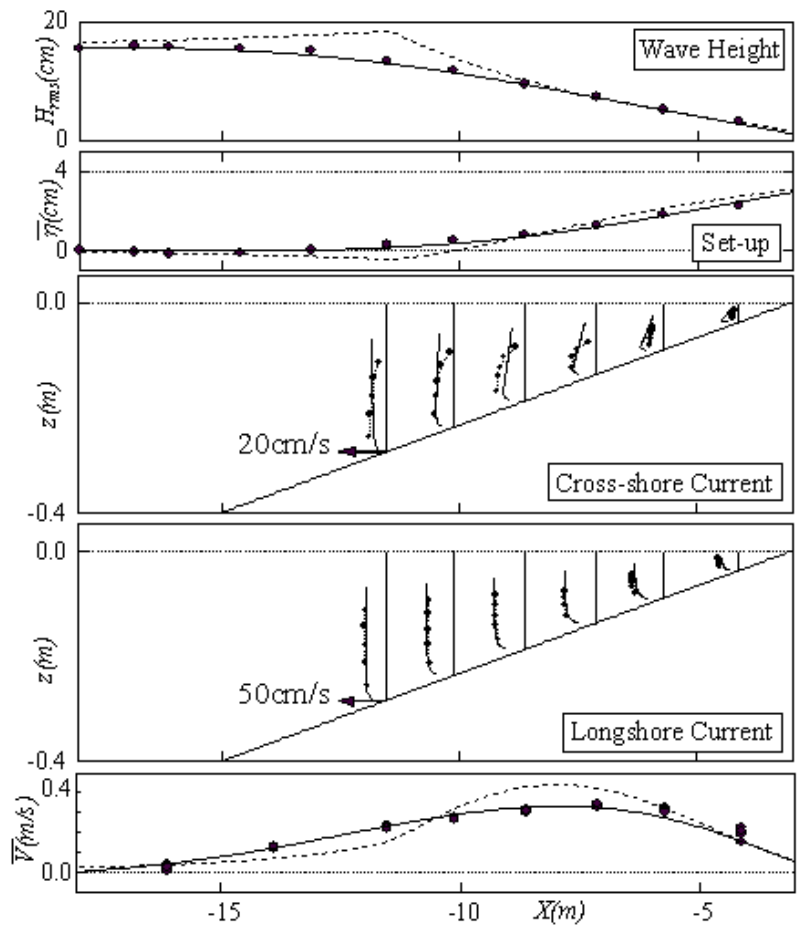

Figure 3. Comparisons of computed wave heights, setup, vertical profiles of cross-shore and longshore current velocities, cross-shore distributions of depth-averaged longshore current velocities with measured data presented by Hamilton and Ebersole (2001) (Test 8A-E, random wave case).

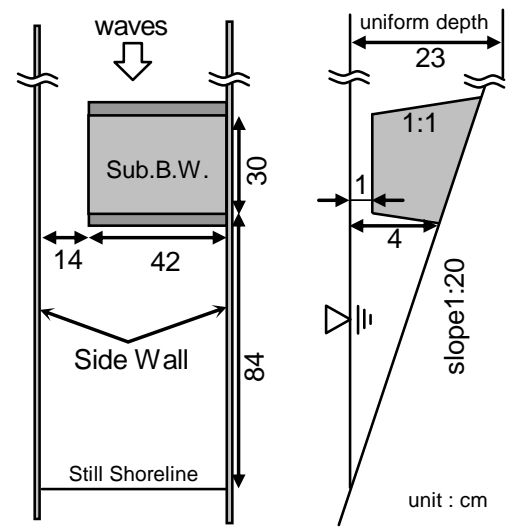

Figure 4. Overview of the experimental setup.

$$
\begin{gathered}
S_{i j}=\frac{1}{2} \overline{\zeta^{2}} \delta_{i j}+\int_{-h}^{0} \overline{u_{i} u_{j}-w^{2} \delta_{i j}} d z \\
q_{w i}=\overline{\int_{0}^{\zeta} u_{s, i} d z}=\overline{P_{i} \zeta} \frac{k}{\tanh k h}
\end{gathered}
$$

where $k$ is wave number determined from wave period and local water depth.

Following Tajima and Madsen(2006), evolutions and dissipations of the surface roller is determined through the energy balance equation of the surface roller. Recalling the conceptual assumption that a part of broken wave dissipation energy should be provided to the growth of the surface rollers, the present model determined balance equation of the surface roller energy, $E_{s}$, by 


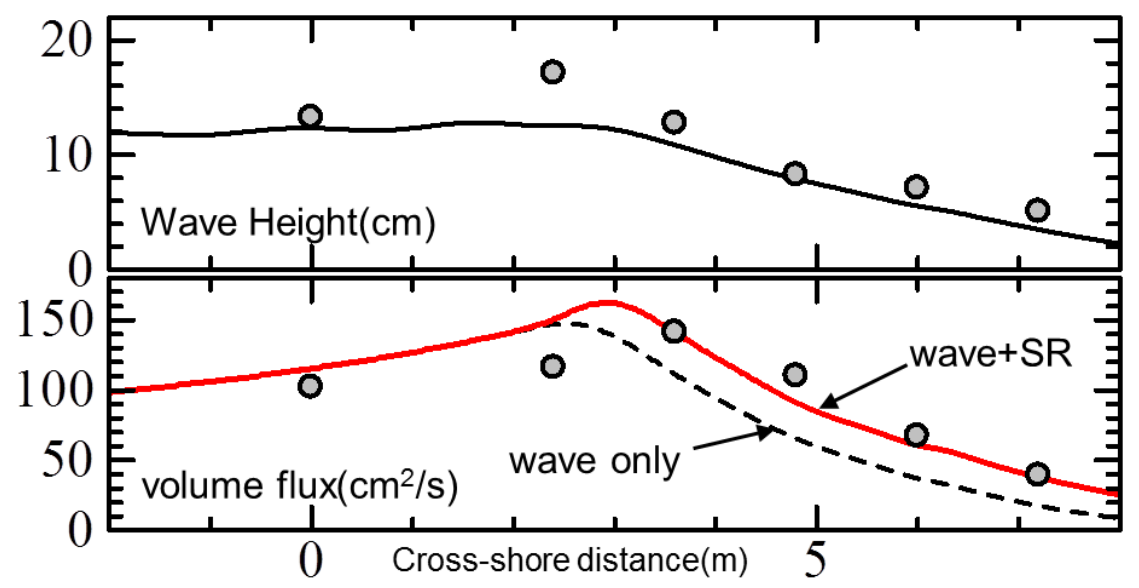

Figure 5 Cross-shore distributions of computed wave heights and shoreward volume flux due to wave and surface roller (SR) compared with measured data (circles) presented by Cox and Kobayashi (1996)

$$
\frac{\partial E_{s r}}{\partial t}+\frac{\partial\left(E_{s r} C n_{i}\right)}{\partial x_{i}}=-\frac{K_{s r}}{h} E_{s r} C+\frac{\partial}{\partial x_{i}}\left(v_{B} \frac{\partial P_{i}}{\partial x_{i}}\right) P_{i}
$$

where $K_{s r}$ is coefficient of the energy dissipation of the surface roller and the second term in the right hand side of (9) is the energy production term, which corresponds to the instantaneous energy dissipation rate of the broken waves. Dispersion coefficient of broken wave, $v_{\mathrm{B}}$, is determined so that the phase averaged broken wave energy dissipation rate corresponds to the that of Tajima and Madsen's (2006) broken wave model based on the energy balance equations. In the numerical model, instantaneous surface roller energy computed from (9) was stored and the present Q3D model applied the phase-averaged value of these obtained instantaneous $E_{s r}$. Figure 5 compares predicted and measured wave heights and shoreward volume flux due to waves and rollers. Measured data were obtained by Cox and Kobayashi (1996) in their one-dimensional flume experiment. In the figure, observed total volume flux was inversely estimated by vertically integrating measured undertow velocity under the wave trough level. As seen in the figure, the present model well explains the wellknown feature that wave-associated volume flux tends to underestimate the volume flux inside the surf zone and the gap between measured and predicted volume flux inside the surf zone is reasonably compensated by the volume flux due to surface roller.

Finally, the entire model was applied to Tajima et al.'s (2007) experimental case with incident wave conditions of $H=3 \mathrm{~cm}$ and $T=0.8 \mathrm{~s}$. This is the case that Boussinesq model could not sufficiently achieve the excellent predictive skills of circulation current patterns behind the detached submerged breakwater (Tajima and Madsen, 2007). Figure 6 compares predicted and measured wave heights and mean current velocity fields. In the figure, three different models were applied for their comparisons: (i) Boussinesq equations with anisotropic dispersion-type broken wave model (Tajima et al., 2007); (ii) the present Q3D model; and (iii) conventional 2DH neashore current model. For comparisons, both Q3D and 2DH models input the same radiation stress obtained from the computations based on the mild slope equations. As seen in the comparisons of the wave heights, linear-based mild slope equation tends to underestimate the wave heights around the breaking point. It should also be pointed out that the present mild slope equation model also underestimates the concentration of the wave heights along the left vertical wall where is located at the gap of submerged breakwaters in the mirror images and thus strong return flow should be dominant. This study did not account for wave-current interaction in the wave model. Except these two features, the present model showed overall good predictive skills of the broken wave height especially behind the breakwater.

As pointed out by Tajima et al. (2007), dispersion-type broken wave model tends to over-smooth mean current components and thus the model tends to underestimate the velocity of horizontal circulating current. Moreover, Boussinesq model does not express excess amount of shoreward 
volume flux due to surface rollers and thus the model requires larger shoreward mean current velocity to keep the same amount of shoreward volume flux as the ones of the Q3D model. This may be another reason why, compared to the measured data, the location of the circulation was shifted toward the shore in the case of Boussinesq model. Similar to the Boussinesq model, 2DH model has stronger shoreward current velocity on the submerged break water and fails to represent the circulating current pattern. In contrast to these models, Q3D model tends to have smaller shoreward current velocity above the submerged breakwater and successfully represent the anti-clockwise circulating current pattern. It should also be pointed out that measured anti-clockwise circulating current tends to have larger offshore-ward current velocity rather than shoreward current velocity. This asymmetric feature is also observed in the predicted current patterns of the present Q3D model. This should be because the model requires larger seaward return flow velocity to compensate the volume flux due to the surface roller.

\section{CONCLUSIOS}

Tajima and Madsen's (2006) undertow model was extended for predictions of mean shear current field on the arbitrary bathymetry. The present model was applied to experimental cases and showed excellent predictive skills of mean current components. One of the most important difference between
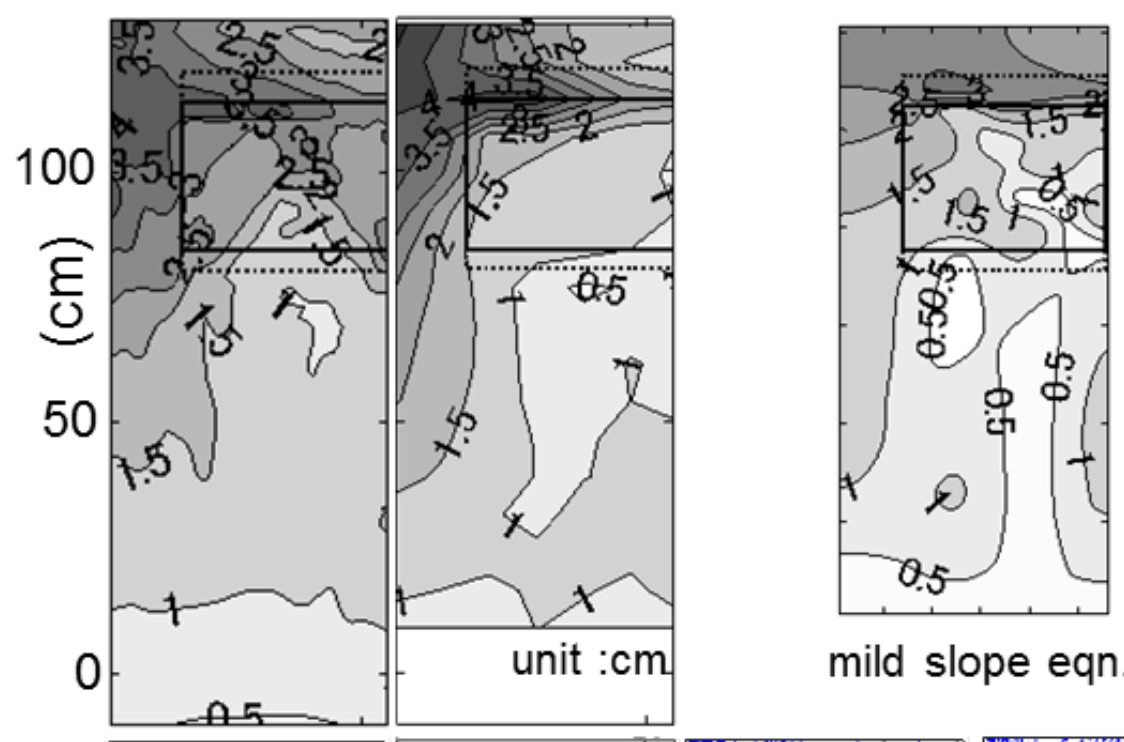

mild slope eqn.

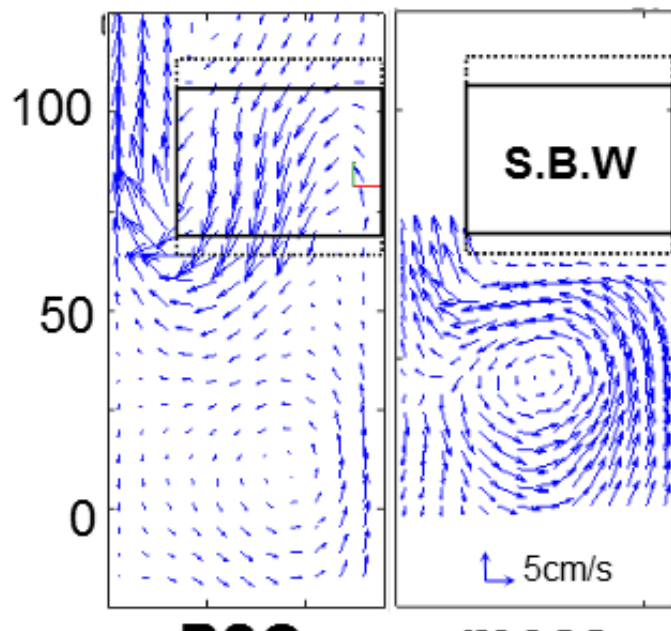

BSQ meas.

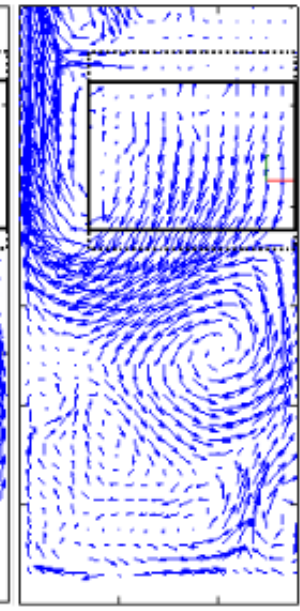

Q3D

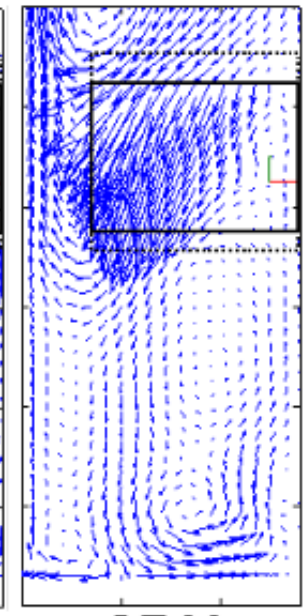

2DH

Figure 6 Comparisons of predicted and measured wave heights and mean current behind the submerged detached breakwater. Predictions are: Boussinesq model (BSQ); the present model (Q3D); and conventional 2DH model without surface roller (2DH). 
Q3D and 2DH models is in that Q3D is able to account for excess amount of shoreward volume flux due to waves and surface rollers while $2 \mathrm{DH}$ is not. Influence of this difference was clearly seen in the predictions of circulating current patterns around the detached submerged breakwater. Further investigation should be performed to examine the more general validity and limitations of the present model.

\section{ACKNOWLEDGMENTS}

This study was gratefully supported by Grant-in-Aid for Young Scientists (A), No. 23686070, from Japan Society for the Promotion of Science (JSPS).

\section{REFERENCES}

Cox, D.T. and N. Kobayashi. 1996. Undertow profiles in the bottom boundary layer under breaking waves, Proc. 25th Int. Conf. on Coast. Eng., ASCE, 3194-3206.

Hamilton, D. G. and Ebersole, B. A. 2001. Establishing uniform longshore currents in a large-scale laboratory facility, Coastal Eng., 42, 199-218.

Kuroiwa, M., H. Noda and Y. Houchi. 1997. A quasi-three dimensional model of wave-induced current in the surf zone, Proc. of Coast. Eng., JSCE, vol.44, 151-155 (in Japanese).

Madsen, O. S. 1994. Spectral wave-current bottom boundary layer flows, Proc. 24th Int. Conf. on Coast. Eng., ASCE, 384-398.

Ohnaka, S., A. Watanabe and M. Isobe. 1989. Numerical computation of wave field by the unsteady mild slope equation extended to wave-current coexisting field, Proc. of Coast. Eng., JSCE, 91-95 (in Japanese).

Putrevu, U., and Svendsen, I. A. 1992. A mixing mechanism in the nearshore region, Proc. 23rd Int. Conf. on Coast. Eng., ASCE, 2758-2771

Sanchez-Arcilla, A., Collado, F., and A. Rodriguez. 1992. Vertically varying velocity field in Q-3D nearshore circulation, Proc. 23rd Int. Conf. on Coastal Eng. 2811-2824.

Svendsen, I.A. and U. Putrevu. 1994. Nearshore mixing and dispersion, Proc. Math. and Phys. Sciences, vol.445, 561-576.

Tajima, Y., and O. S. Madsen. 2006. Modeling near-shore waves, surface rollers, and undertow velocity profiles, J. of Waterway, Port, Coastal and Ocean Eng., Vol. 132, No. 6, 429-438.

Tajima, Y. and S. Sato. 2010. Local concentration of slowly varying wave and current fields around the abruptly changing bottom slopes along the shore, Proc. 32nd Int. Conf. Coast. Eng.,

Tajima, Y., S. Sato, T. Shimozono and M. Isobe. 2007. Modeling of wave-induced current around submerged detached breakwaters, Proc. of Int. Conf. on Coast. Str. 2007, CD-ROM published by World Scientific Publishing Corp. and East Meets West Productions. 